\title{
NANOINDENTATION OF HYDROGEN ENRICHED Zr-1Nb ZIRCONIUM ALLOY NUCLEAR FUEL CLADDINGS
}

\author{
Ondřej Libera ${ }^{a, *}$, Patricie Halodová $^{a}$, Petra Gávelová ${ }^{a}$, \\ JAKUB KREJČÍ ${ }^{b}$
}

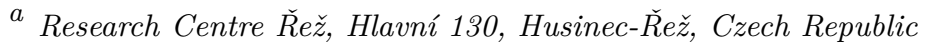

${ }^{b}$ UJP Praha a.s, Nad Kamínkou 1345, Praha-Zbraslav, Czech Republic

* corresponding author: ondrej.libera@cvrez.cz

\begin{abstract}
Zirconium alloys are being commonly used as a material of choice for nuclear fuel claddings in water cooled nuclear reactors for decades due to their good corrosion resistance and low neutron absorption. However, the increasing operation conditions of next generation nuclear reactors (Gen-IV) in terms of higher temperatures, pressures and higher neutron flux requires evaluation of further $\mathrm{Zr}$ cladding usability. The embrittlement of $\mathrm{Zr}$ claddings due to hydrogen pickup from reactor coolant is one of the issues for its potential use in Gen-IV reactors. Nanoindentation is an effective tool for analysis of the change of mechanical properties of hydrogen enriched $\mathrm{Zr}$ claddings from localised material volume. Zirconium alloy Zr-1Nb (E110) with experimentally induced hydrides was analysed by the means of nanoindentation. Zirconium hydrides were formed in the material after exposure in high temperature water autoclave. The optimized methodology of surface preparation suitable for nanoindentation is described and the resulting surface quality is discussed. The nanoindentation measurements were performed as an array of 10x10 indents across areas with hydrides. Depth dependent hardness and reduced modulus values measured by nanoindentation were compared between the material with no hydrogen content, low hydrogen content (127 ppm H) and high hydrogen content (397 ppm H). Complementary microhardness measurements at HV 0.1 were performed on all materials for bulk material hardness comparison.
\end{abstract}

KEYWORDS: Nanoindentation, nuclear fuel claddings, Zr-1Nb.

\section{INTRODUCTION}

The nuclear fuel cladding serves as the first containment barrier of the fuel pellets in water-cooled nuclear reactors and subsequently in spent fuel storage casks. Due to their good mechanical properties, corrosion resistance and low neutron absorption, zirconium alloys are being commercially used as a nuclear fuel cladding material for decades. Despite all the benefits, zirconium claddings chemically react with the cooling water in the reactor, which results in creation of hydrides in the material volume [1. Hydrides formed in zirconium claddings affect the mechanical properties of the material, making it prone to cracking due to the internal stresses caused by fuel swelling and the propagation of fission gasses during the nuclear fuel cycle. This process of cracking associated with hydrides in $\mathrm{Zr}$ alloys is called Delayed Hydride Cracking (DHC) [2] This phenomenon is one of the key issues with use of Zr alloy claddings in Gen-IV nuclear reactors.

Nanoindentation is one of the analytical methods to evaluate the change of mechanical properties before and after hydridation in a very local volume of the material. The mechanical properties of various hydrogen enriched $\mathrm{Zr}$ claddings [3, 4] and pure Zr [5] have already been assessed by nanoindentation measurements in previous studies. However, most of the research in the field was performed on western type on Zr claddings such as Zircalloy and Zr-2.5Nb. The amount of available research articles published on nanoindentation of E110 alloy in English is very limited to non-existent, despite the fact that this type of cladding is still being actively used in a large part of the world.

In this work, nanoindentation testing was performed on $\mathrm{Zr}-1 \mathrm{Nb}$ (E110) zirconium alloy nuclear fuel claddings. The tests were conducted on reference and artificially hydrided claddings for future comparison with material irradiated in real operating conditions in pressurised water nuclear reactor (PWR). Measurements were performed across the areas containing hydrides. The comparison of mechanical properties between claddings with various amount of hydrogen content is assessed.

\section{EXPERIMENTAL}

\subsection{MATERIAL}

The material subjected for testing was E110 zirconium alloy cladding tube (Zr, 1 wt.\% Nb, 0.5 wt.\% $\mathrm{Fe}, 0.1$ wt.\% O) in reference state and two hydrogen enriched states. Two cladding tubes were exposed to $425^{\circ} \mathrm{C}$ pressurised deionized water at $10.7 \mathrm{MPa}$ in an autoclave for 63 and 243 days. Hydrogen content in samples was evaluated by inert gas fusion (IGF) technique and was estimated as $122 \mathrm{ppm}$ and $397 \mathrm{ppm}$ [6]. 


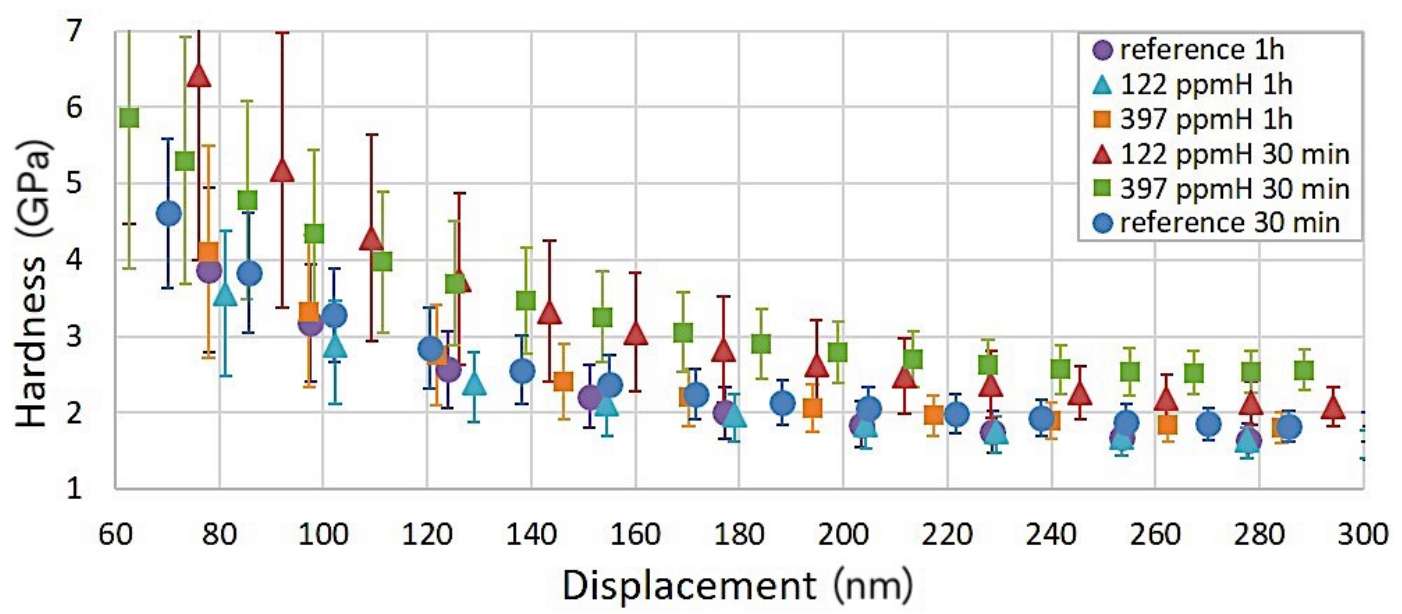

Figure 1. Comparison of average hardness values of samples finished by chemo-mechanical polishing for 30 minutes and 1 hour.

The final concentration of hydrogen in samples approximately represents the state of cladding during the active use in PWR (122 ppm $\mathrm{H})$ and during dry storage at the end of nuclear fuel cycle (397 ppm H). One milimeter thick rings were cut from each cladding tube on Buehler IsoMet gravitational saw with diamond cutting disc. Cut rings were heat embedded in Condufast (Struers) conductive polymer resin and ground on automatic grinding machine Struers Tegramin-25 using sandpapers with 500-2000 grits. The samples were then polished on the same machine with 3 and $1 \mu \mathrm{m}$ diamond suspensions and finished with $0.05 \mu \mathrm{m}$ colloidal silica suspension OPS (Struers). The final polishing step was performed on a fine polishing cloth with a mixture of $0.05 \mu \mathrm{m}$ colloidal silica suspension OPS with $\mathrm{H}_{2} \mathrm{O}_{2}$ (30\%vol.) and $\mathrm{HF}$ (40\% weight) in 50:10:1 vol. proportion [4]. This chemo-mechanical polishing combination of polishing suspension and etching solution was essential for the preparation of damage-free surface with excellent visibility of the microstructure containing hydrides. Commonly used chemical or electrolytic polishing methods for microstructure enhancement and reduction of mechanically hardened layer [7] proved inappropriate for nanoindentation measurements. The inhomogeneous etching rate of Zr matrix and hydride phases during these processes introduces increased surface roughness and etch pits, which might compromise the validity of nanoindentation data.

\subsection{INDENTATION TESTING}

Nanoindentation tests were performed on Bruker/Hysitron Ti 950 triboindenter. Diamond Berkovich type indenter tips were used for all measurements. Calibration of tip area function [8] for hardness and reduced modulus calculations were performed on fused silica calibration standard from Hysitron $(\mathrm{Er}=69.6 \mathrm{GPa} ; \mathrm{H}=9.25 \mathrm{GPa})$. Nanoindentation measurements on E110 samples were performed across the area with visible hydrides as a 10x10 indentation matrix with $20 \mu \mathrm{m}$ separation between indents. Partial unload load function [9] with gradual increase in maximum load up to $10 \mathrm{mN}$ was selected to obtain various depth dependent results for each indentation measurement. Hardness and reduced modulus values were calculated from the nanoindentation load displacement curves utilizing the Oliver\&Pharr analytical model 8]. Microhardness measurements on Struers DuraScan were performed with diamond tip for hardness comparison at high indentation depths. An array of 10 indents with $100 \mu \mathrm{m}$ spacing was performed around the hoop direction in the central part of the ring of each sample at HV 0.1 with Vickers diamond tip.

\section{Results AND Discussion}

\subsection{SAMPLE PREPARATION}

The duration of chemo-mechanical polishing surface finishing step was found to be crucial for correct nanoindentation measurement on E110 samples. Despite the good microstructure visibility after 30 minutes of surface finish, the chemical-mechanical polishing did not completely remove the mechanically hardened layer formed by mechanical grinding and polishing. Figure 1 shows the comparison of the measured nanoindentation hardness between chemomechanically polished samples prepared for 30 minutes and 1 hour. The effect of nano-crystallic hardened layer on mechanical properties is best visible on approx. 0.5 GPa increase in overall hardness between hydrogen enriched samples in both states.

Indentation results presented and discussed later in this work (see section 3.2 and further) are from samples with $1 \mathrm{~h}$ of surface finish, where the mechanically hardened layer has been reduced by proper sample preparation.

\subsection{IndENTATION TESTING RESUlts}

The area for nanoindentation measurement on 122 ppm $\mathrm{H}$ sample was selected to be around the area 


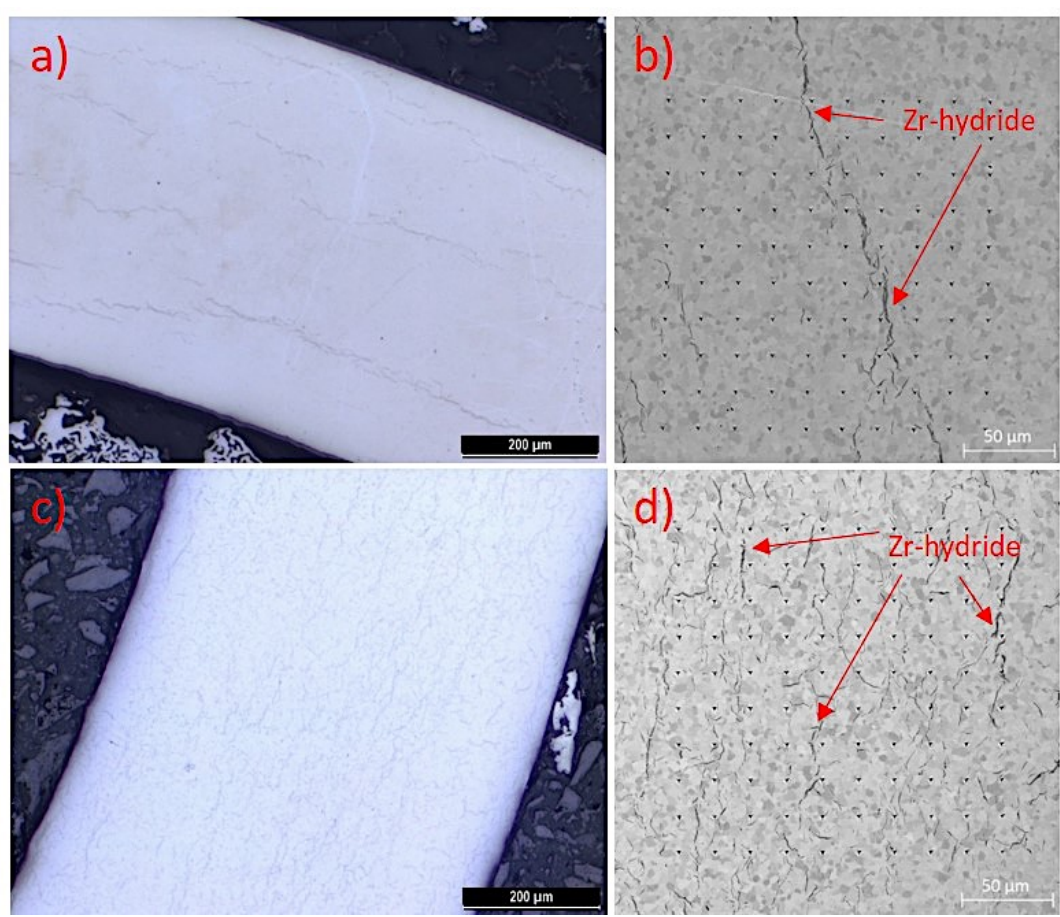

FIgURE 2. a) Light optical micrograph of hydride distribution in a sample with $122 \mathrm{ppm} \mathrm{H.} \mathrm{b)} \mathrm{BSE} \mathrm{image} \mathrm{of}$ indentation matrix in area with hydride on $122 \mathrm{ppm} \mathrm{H}$ sample. c) Light optical micrograph of hydride distribution in a sample with 397 ppm H. d) BSE image of indentation matrix on 397 ppm H sample.

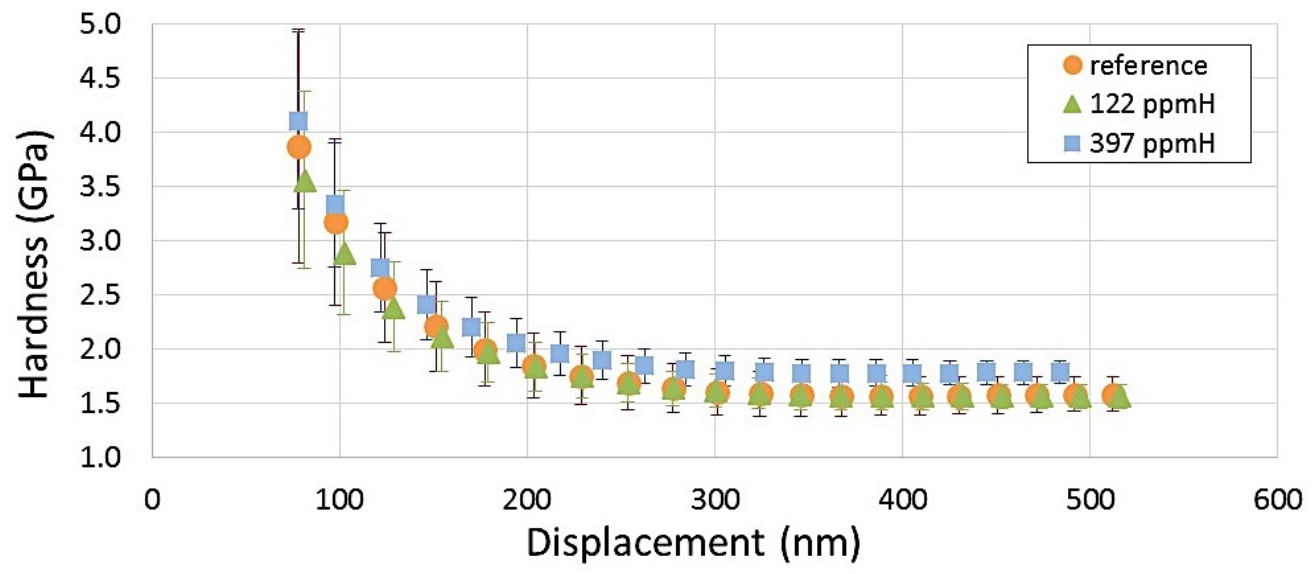

FigurE 3. Comparison of average hardness values of reference, $122 \mathrm{ppm}$ and $397 \mathrm{ppm}$ hydrogen enriched E110 samples.

\begin{tabular}{lccc}
\hline & E110 Reference & E110 122 ppm H & E110 397 ppm H \\
\hline H 400nm (GPa) & $1.56 \pm 0.18$ & $1.56 \pm 0.12$ & $1.77 \pm 0.16$ \\
Er 400nm (GPa) & $72.75 \pm 7.63$ & $72.92 \pm 2.56$ & $78.14 \pm 3.24$ \\
HV 0.1 (GPa) & $1.44 \pm 0.06$ & $1.44 \pm 0.05$ & $2.09 \pm 0.18$ \\
\hline
\end{tabular}

TABLE 1. Nanoindentation hardness and reduced modulus at $400 \mathrm{~nm}$ indentation depth and microhardness (HV 0.1) results for all samples.

with hydride from LOM observation shown on figure $2 \mathrm{a}$. At this level of hydrogen pick up the hydrides were formed as individual chains in the hoop direction of the ring. The spacing between individual hydride chains was large, approximately $1 / 4$ of the ring width. For this reason, there was only one major hydride chain reached in the nanoindentation matrix (figure 2p). The light and dark stains in BSE images represent individual grains with various grain orientations. The hydrides on $397 \mathrm{ppm} \mathrm{H}$ sample were much shorter and distributed evenly across the whole area of the ring. The orientation of larger 397 ppm H E110 hydrides was mainly in the hoop direction with minor hydrides in radial direction shown on Figure 2k. The 


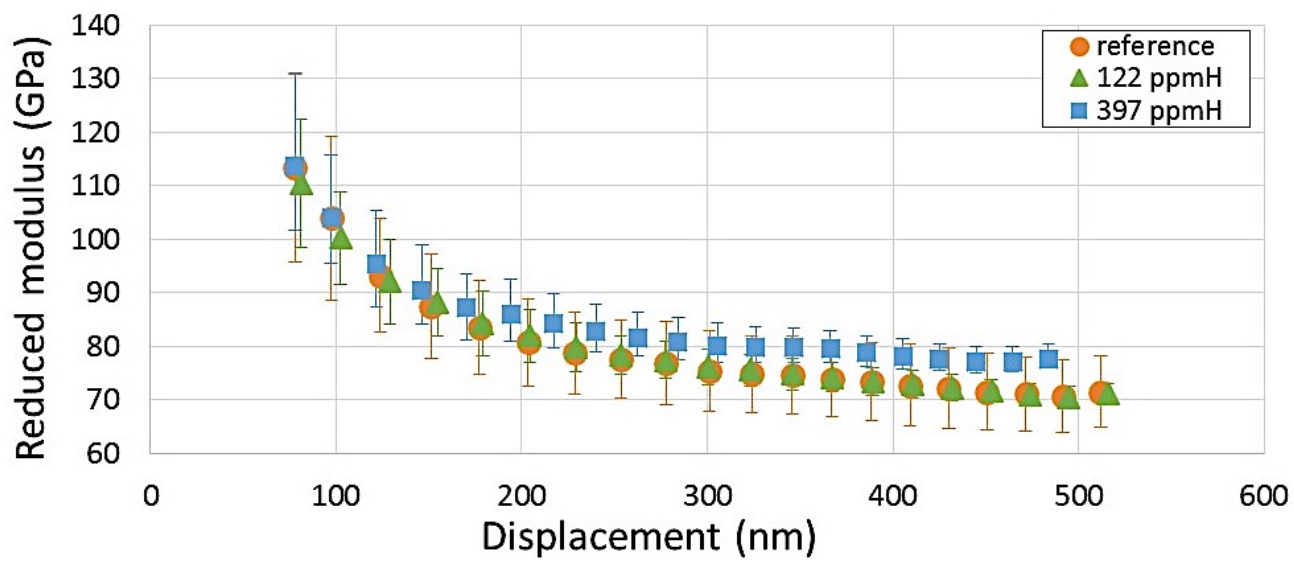

Figure 4. Comparison of average reduced modulus values of reference, $122 \mathrm{ppm}$ and $397 \mathrm{ppm}$ hydrogen enriched E110 samples.

indentation area on $397 \mathrm{ppm} \mathrm{H}$ sample was placed in the middle of the ring, covering the representative area with hydrides (Figure 2 $\mathrm{d}$ ). The indentation matrix on reference sample was placed in the central part of the ring. The hardness vs. displacement results from nanoindentation measurements plotted in Figure 3 show the overall hardness profile across all measured depths for all studied samples. Results of the same measurements are shown in Figure 4 for reduced modulus values. It is apparent that hardness measurements on all samples were affected by the indentation size effect (ISE), causing the increase in hardness values in shallow indentation depths. This effect is well known and unavoidable at these indentation depths [10]. A model for estimation of correct hardness values at low indentation depths was proposed by Nix \& Gao [11, however its application is not in the scope of this work. The increased reduced modulus at low indentation depths $(<200 \mathrm{~nm})$ might be caused by a thin oxide layer formed after sample preparation. This assumption has to be confirmed by further investigation of the surface after the preparation process. An increase of hardness and reduced modulus is visible at 397 ppm H sample. A dense mesh of hydrides in the material directly affects the mechanical properties of the whole material. Measured mechanical properties of $122 \mathrm{ppm} \mathrm{H}$ sample are nearly identical to values from the reference sample. It can be assumed that the scarce presence of hydrides in $122 \mathrm{ppm} \mathrm{H}$ sample affects the mechanical properties of the whole material just slightly. However, a closer examination of the mechanical properties near the hydride has to be performed to confirm this assumption.

Comparison of hardness measured by nanoindentation and HV 0.1 microhardness is shown in Table 1. The value of $400 \mathrm{~nm}$ was selected as a stable nanoindentation depth without the influence of the ISE for comparison. A good agreement of measured values is shown for reference E110 and 122 ppm H sample. The microhardness results for 397 ppm H show approx. $0.3 \mathrm{GPa}$ higher value than the nanoindentation measurement. The reason behind this difference is a matter of further research.

\section{Conclusions}

Optimized sample preparation method was used for fine surface preparation of E110 samples for nanoindentation measurements. Samples of Zr-1Nb E110 nuclear cladding tubes in reference, $122 \mathrm{ppm}$ and 397 ppm hydrogen enriched states were measured by nanoindentation. It was shown that hardness and reduced modulus values of the $122 \mathrm{ppm} \mathrm{H}$ sample were very close to the reference sample. This result was confirmed by comparison to microhardness testing results. A further research needs to be performed closer to hydride chains on $122 \mathrm{ppm} \mathrm{H}$ sample to confirm the preservation of mechanical properties despite the hydrogen build-up. The measurements on 397 ppm $\mathrm{H}$ sample show an increase of hardness by approx. $13 \%$ and reduced modulus increase by $8 \%$. The measured microhardness value was $18 \%$ higher than nanoindentation hardness measured at $400 \mathrm{~nm}$. This phenomenon is a subject of further research.

\section{ACKNOWLEDGEMENTS}

The presented work was financially supported by the Ministry of Education, Youth and Sport Czech Republic Project LQ1603 (Research for SUSEN). This work has been realized within the SUSEN Project (established in the framework of the European Regional Development Fund (ERDF) in project CZ.1.05/2.1.00/03.0108).

\section{REFERENCES}

[1] C. Coleman, D. Hardie. The hydrogen embrittlement of $\alpha$-zirconium-A review. Journal of the Less Common Metals 11(3):168 - 185, 1966. DOI:10.1016/0022-5088(66)90003-8

[2] Delayed hydride cracking in zirconium alloys in pressure tube nuclear reactors. final report of a coordinated research project 1998-2002. Tech. Rep. 41, International Atomic Energy Agency, Nuclear Power Technology Development Section, Vienna (Austria), 2004. 
[3] K. Kese, P. Olsson, A.-M. A. Holston, E. Broitman. High temperature nanoindentation hardness and young's modulus measurement in a neutron-irradiated fuel cladding material. Journal of Nuclear Materials 487:113 - 120, 2017. DOI:10.1016/j.jnucmat.2017.02.014

[4] A. Rico, M. Martin-Rengel, J. Ruiz-Hervias, et al. Nanoindentation measurements of the mechanical properties of zirconium matrix and hydrides in unirradiated pre-hydrided nuclear fuel cladding. Journal of Nuclear Materials 452(1):69 - 76, 2014. DOI:10.1016/j.jnucmat.2014.04.045

[5] M. Kuroda, D. Setoyama, M. Uno, S. Yamanaka. Nanoindentation studies of zirconium hydride. Journal of Alloys and Compounds 368(1):211 - 214, 2004. DOI:10.1016/j.jallcom.2003.08.094.

[6] B. D. Holt, H. T. Goodspeed. Determination of nitrogen, oxygen, and hydrogen in metals by inert gas fusion. a manometric method. Analytical Chemistry 35(10):1510-1513, 1963. DOI:10.1021/ac60203a023.

[7] G. Finch, A. Quarrell. The beilby layer. Nature Publishing Group 137(3467):516-519, 1936. DOI:10.1038/137516a0
[8] W. Oliver, G. Pharr. An improved technique for determining hardness and elastic modulus using load and displacement sensing indentation experiments. Journal of Materials Research 7(6):1564-1583, 1992. DOI:10.1557/JMR.1992.1564

[9] A. C. Fischer-Cripps. Nanoindentation. Springer, New York, NY, 3rd edn., 2011. DOI:10.1007/978-1-4419-9872-9

[10] Y. Milman, A. Golubenko, S. Dub. Indentation size effect in nanohardness. Acta Materialia 59(20):7480 7487, 2011. DOI:10.1016/j.actamat.2011.08.027

[11] W. D. Nix, H. Gao. Indentation size effects in crystalline materials: A law for strain gradient plasticity. Journal of the Mechanics and Physics of Solids 46(3):411 - 425, 1998. DOI:10.1016/S0022-5096(97)00086-0. 